\title{
Anomalous Heat Conduction in One-Dimensional Momentum-Conserving Systems
}

\author{
Onuttom Narayan ${ }^{1,2}$ and Sriram Ramaswamy ${ }^{1}$ \\ ${ }^{1}$ Centre for Condensed Matter Theory, Department of Physics, Indian Institute of Science, Bangalore 560012, India \\ ${ }^{2}$ Department of Physics, University of California, Santa Cruz, California 95064*
}

\begin{abstract}
We show that for one-dimensional fluids the thermal conductivity generically diverges with system size $L$ as $L^{1 / 3}$, as a result of momentum conservation. Our results are consistent with the largest-scale numerical studies of two-component hard-particle systems. We suggest explanations for the apparent disagreement with studies on Fermi-Pasta-Ulam chains.
\end{abstract}

When a very small temperature difference is applied across a system, it is expected that in steady state the heat current $j$ will obey Fourier's law of conduction

$$
j=-\kappa \nabla T,
$$

where $T$ is the local temperature and $\kappa$ is the heat conductivity of the material. Although $\kappa$ is in general temperature dependent, if the applied temperature difference is small it should be constant across the system. Thus if $T_{1}$ and $T_{2}$ are the temperatures at which the two ends of a system of length $L$ are kept (with $T_{1} \geq T_{2}$ ), the steady state current should be $j=\kappa\left(T_{1}-T_{2}\right) / L$.

On the other hand, for many one-dimensional models [1] it can be shown analytically [2-4] or numerically [4-8] that $j \propto L^{\alpha-1}$ with $\alpha>0$, even in the linear response regime where $j \propto T_{1}-T_{2}$. The value of $\alpha$ differs from model to model. In the framework of Eq. (1), this would imply an $L$-dependent conductivity that diverges in the infinite system limit. (In some oscillator models, $\alpha<0$, implying an anomalous but not divergent conductivity [3].)

Recently, it has been argued [9] that such anomalous heat conduction occurs only in systems with momentum conservation, and is a consequence thereof. This was done by showing that if $j(x, t)$ is the energy current density, the autocorrelation function of the total energy current $J(t)=$ $\int d x j(x, t)$

$$
C\left(t_{1}-t_{2}\right)=\left\langle J\left(t_{1}\right) J\left(t_{2}\right)\right\rangle
$$

has the property $C(t \rightarrow \infty) \neq 0$. Although the proof of this result in Ref. [9] was specific to one-dimensional systems, Galilean invariance allows one to construct a general proof for any dimension $d$ [10]. This is because the energy current $J(t)$ has an advective contribution $\left(E+p L^{d}\right) \bar{v}$, where $E$ is the energy, $p$ the pressure, $L^{d}$ the volume, and $\bar{v}$ the center of mass velocity of the system. For an energy and momentum conserving system, this advective contribution to $J(t)$ is time independent. $C(\infty)$ is found by calculating $\left\langle\left[\left(E+p L^{d}\right) \bar{v}\right]^{2}\right\rangle$ within the canonical ensemble, and is nonzero. The limit $\lim _{\tau \rightarrow \infty} \lim _{L \rightarrow \infty}\left[1 /\left(T^{2} L\right)\right] \int_{0}^{\tau} d t C(t)$ is then divergent. The Kubo formula [11] was invoked [9] to equate this to $\kappa$.
Although (as discussed in the next paragraph) the argument in Ref. [9] is incorrect and a nonvanishing $C(\infty)$ has no consequence for heat conduction, the conclusion that momentum conservation in low-dimensional systems (generically) implies anomalous conduction is valid. This is because, in addition to the limit $C(\infty)$ being finite, there is also a slowly decaying tail in $C_{0}(t)=C(t)-$ $C(\infty)$. Unlike $C(\infty) \neq 0$, which is valid in all dimensions, the tail in $C_{0}(t)$ decays sufficiently slowly to cause a singularity in $\kappa$ only for $d \leq 2$. In this paper, using the transport equations for a normal fluid with thermal noise added, we show that $\kappa(L) \propto L^{\alpha}$, with $\alpha=(2-d) /$ $(2+d)>0$ for $d<2$. (There is a logarithmic singularity for $d=2$.) For the physical case of $d=1$, we obtain $\alpha=1 / 3$. These transport equations should be valid for all systems which rapidly reach local thermal equilibrium, and for which the only slowly evolving quantities are the mass, energy, and momentum densities. The results are therefore generic, although the assumption of local thermal equilibrium breaks down for some models, such as hard sphere equal mass particles in one dimension, or a chain of harmonic oscillators, for which $\alpha$ is different [2].

We first recall the discussion of Bonetto et al. [12] about the argument in Ref. [9]. The conductivity in momentum conserving systems is in fact not obtained from the autocorrelation function of $J(t)$, but of $J_{0}(t)=J(t)-$ $\left(E+p L^{d}\right) \bar{v}$ [13]. This result can be proved rigorously within linear response theory [14]. If one makes a linearized hydrodynamic approximation, which is equivalent to linear response theory with no (thermal) noise terms in the transport equations, the derivation of this result is simpler and well known [15,16]. When $J(t)$ is replaced by $J_{0}(t)$, the resultant truncated correlation function $C_{0}(t)$ has no infinite time tail, and

$$
\kappa=\lim _{\tau \rightarrow \infty} \lim _{L \rightarrow \infty} \frac{1}{T^{2} L} \int_{0}^{\tau} d t C_{0}(t)
$$

is cured of its divergence.

Despite the fact that $C_{0}(\infty)=0$, we show in this paper that, with thermal noise in the transport equations, $C_{0}(t)$ decays sufficiently slowly with $t$ to make the integral $\int d t C_{0}(t)$ - and therefore the conductivity $\kappa$ - divergent 
for $d \leq 2$. We first show this qualitatively. The advective contribution to $J(t)$ is really equal to $\int d^{d} x h(x) v(x)$, where $h(x)$ is the local enthalpy density and $v(x)$ the local velocity. (Vector indices have been suppressed.) This can be expressed as $\int d^{d} k h(k) v(-k)+\left(E+p L^{d}\right) \bar{v}$, where the first integral is restricted to $k \neq 0$, and the second part comes from the nonzero spatial average of $h(x)$. In going from $J(t)$ to $J_{0}(t)$, only the second part of this was removed. Since the time decay of all the hydrodynamic modes is diffusive [18], expressing $h(k, t)$ and $v(k, t)$ in terms of hydrodynamic modes and approximating $\left\langle h(k, t) v(-k, t) h\left(k^{\prime}, 0\right) v\left(-k^{\prime}, 0\right)\right\rangle$ as $\left\langle h(k, t) h\left(k^{\prime}, 0\right)\right\rangle \times$ $\left\langle v(-k, t) v\left(-k^{\prime}, 0\right)\right\rangle$ yields the advective contribution to $C_{0}(t)$ to be $\sim L^{d} \int d^{d} k \exp \left[-O\left(k^{2}\right) t\right]$, which is $\sim L^{d} / t^{d / 2}$. From Eq. (3), $\kappa$ diverges for $d \leq 2$ [19].

Although the conductivity $\kappa$ does indeed diverge for $d \leq 2$ as this rough calculation indicates, the tail of $C_{0}(t)$ decays as $t^{-2 d /(d+2)}$ instead of as $t^{-d / 2}$. This is because thermal noise in the transport equations gives rise to singular corrections to the parameters in the equations, so that the hydrodynamic modes decay superdiffusively. As is standard in renormalization group (RG) analyses of such phenomena, we solve the linearized transport equations and check whether the nonlinear corrections are relevant for long wavelength low frequency phenomena. Below $d=2$, which is thus the upper critical dimension, the nonlinearities are found to be relevant, and their effect is calculated.

We assume that the system whose thermal conductivity is of interest is one which reaches local thermal equilibrium, with the only dynamical variables that evolve slowly with time being the mass, energy, and momentum densities. With these assumptions, the appropriate transport equations for the system are those for a normal fluid $[18,20]$, with thermal fluctuations included in the form of noise sources $[21,22]$ :

$$
\begin{aligned}
\partial_{t} \rho+\nabla \cdot(\rho v)= & 0, \\
\partial_{t}(\rho v)+\nabla \cdot(\rho v v)= & -\nabla p+[\zeta+\eta(d-2) / d] \nabla \nabla \cdot v \\
& +\eta \nabla^{2} v+\zeta_{v}, \\
\partial_{t} \epsilon+\nabla \cdot[(\epsilon+p) v]= & \nabla \cdot \kappa_{0} \nabla T+O\left[(\nabla v)^{2}\right]+\zeta_{\epsilon},
\end{aligned}
$$

where $\rho$ is the local density of the fluid. The local temperature $T$ and pressure $p$ are implicit functions of $\rho, v$, and $\epsilon$. The thermal noise terms $\zeta_{v, \epsilon}$ satisfy $\left\langle\zeta_{v}\left(x_{1}, t_{1}\right) \zeta_{v}\left(x_{2}, t_{2}\right)\right\rangle \propto-k_{B} T \delta\left(t_{1}-t_{2}\right) \partial^{2} \delta\left(x_{1}-x_{2}\right)$, and similarly for $\zeta_{\epsilon}$, with the proportionality constants fixed by the requirement that the variance of the fluctuations $\delta \rho=\rho-\rho_{0}, \delta \epsilon=\epsilon-\epsilon_{0}$, and $v$ at any instant are those of a system in equilibrium at temperature $T$. $\left[\nabla \cdot(\rho v v)\right.$ is a vector whose $i$ th component is $\partial_{j}\left(\rho v_{i} v_{j}\right)$.] The first equation in Eqs. (4) is an exact identity, and has no thermal noise correction.

It is standard and straightforward to solve these equations with the linear approximation, valid if $\delta \rho(x, t)$, $v(x, t)$, and $\delta \epsilon(x, t)$ are small. One obtains [15,18] two propagating sound modes and one nonpropagating heat mode. All three modes decay diffusively. The relevance or irrelevance of the nonlinear terms in the transport equations is then determined by calculating corrections to correlation and response functions to one loop in a diagrammatic perturbation expansion. For instance, the response of $v(x)$ to a perturbation in the second member of Eqs. (4), $g_{v v}\left(x_{1}-x_{2} ; t_{1}-t_{2}\right)$, receives a one-loop correction from $\nabla \cdot(\rho v v)$, resulting in corrections to the viscosities $\eta, \zeta$ of the form

$$
\delta \eta, \delta \zeta \sim \int d^{d} x d t c_{v v}(x ; t) g_{v v}(x ; t),
$$

where $c_{v v}$ is the autocorrelation function of $v(x)$. (The component indices in $c_{v_{i}, v_{j}}$ and $g_{v_{i}, v_{j}}$ have been suppressed for compactness.) Expanding the correlation function $c$ and the response function $g$ in terms of the three hydrodynamic modes, and performing the $x$ integral first, the integrand is negligible outside a region of volume $O(t)^{d / 2}$. (The propagating parts of the modes shift the peak of the integrand away from $x=0$ if the contribution of the same hydrodynamic mode is considered for $c$ and $g$. This is inconsequential if the system is large.) Since the correlation and response functions of all the hydrodynamic modes have a $|t|^{-d / 2}$ prefactor, the integral over $x$ yields $\delta \eta, \delta \zeta \sim \int d t \theta(t) t^{-d / 2}$, where the $\theta$ function comes from causality in the response function. The $t$ integral diverges for $d \leq 2$. Similar calculations can be carried out for other one-loop corrections. Since all the autocorrelation functions have the same $|t|^{-d / 2}$ prefactor, the RG scaling dimension of all three of the density fields is $-d / 2:|t|^{-d / 2} \sim\left(|x|^{-d / 2}\right)^{2}$.

For $d \leq 2$, the nonlinear corrections are therefore relevant when expanding around the linearized equations. This can also be seen by scaling all the variables in the transport equations as $x=\lambda x^{\prime}, t=\lambda^{z} t^{\prime}, \zeta_{v, \epsilon}=$ $\lambda^{-(d+2+z) / 2} \zeta_{v, \epsilon}^{\prime}$, and $(\delta \rho, v, \delta \epsilon)=\lambda^{-d / 2}(\delta \rho, v, \delta \epsilon)$. The time derivative, dissipative, and thermal noise terms in Eqs. (4) scale identically if the dynamic exponent $z$ is set to 2. (The terms with one spatial derivative in the linearized equations grow, since they control the propagation of the sound modes, whose speed is obviously altered if $t$ is scaled as $x^{2}$. However, as we have seen in the previous paragraph, this does not affect the scaling of the loop corrections.) The nonlinear terms can be seen to be relevant if $d<2$.

For $d<2$, a renormalization group analysis has to be carried out, integrating out loop corrections from short wavelength fluctuations along with rescaling the variables. The nonlinearities grow under the RG flows until they reach a nontrivial fixed point. The new scaling dimensions of the fields and the dynamic exponent $z$ can then be evaluated at this fixed point. It is, however, not necessary to carry out such a calculation to obtain the exponents: they can be determined completely from symmetry considerations. The RG flows preserve the property that equal time fluctuations in $\delta \rho, v$, and $\delta \epsilon$ must be those of a system in equilibrium at temperature $T$. Since the 
fluctuations in these densities must be Gaussian at sufficiently long wavelength, we require that $\int d^{d} x\left[v^{2},(\delta \rho)^{2}\right.$, $\left.(\delta \epsilon)^{2}\right]$ should be invariant under rescaling. Thus the scaling dimensions of all three fields are equal to $-d / 2$ even for $d<2$. Further, Galilean invariance relates the loop corrections to $\partial_{t} \phi$ and to the corresponding advective term $\nabla \cdot(v \phi)$ for any (conserved) field $\phi$ [23]. Since both terms are invariant at the fixed point, this yields the condition that $\nabla v$ scales as $\partial_{t}$, i.e., $v$ scales as $x / t$. Combining this condition with the previous one, we obtain $z=1+d / 2$.

The energy current density is obtained by requiring that the third equation in Eq. (4) should be equivalent to $\partial_{t} \epsilon+\nabla \cdot j=0$. This yields $j(x, t)=(\epsilon+p) v-$ $\kappa_{0} \nabla T+O\left(\nabla v^{2}, v \nabla \cdot v\right)$. Since $\quad J_{0}(t)=J(t)-(E+$ $\left.p L^{d}\right) \bar{v}$, we obtain correspondingly $j_{0}(x, t)=(\delta \epsilon+p-$ $\left.p_{0}\right) v-\kappa_{0} \nabla T+O\left(\nabla v^{2}, v \nabla \cdot v\right)$. Under the RG rescaling, the three terms in this scale with dimension $-d$, $-1-d / 2$, and $-1-d$, respectively, and the first term is most important for $d<2$. If one expresses the transport equations Eqs. (4) through a generating functional [24], and adds an extra $a(\delta \epsilon+\delta p) v$ term in the argument of the exponent, the $v \rightarrow-v, x \rightarrow-x$ symmetry of Eqs. (4) ensures that this term is not renormalized to $O(a)$. Thus the scaling of $C_{0}(t) / L^{d}$ can be obtained from the bare scaling dimension of $j_{0}(x, t)$ :

$$
C_{0}(t) / L^{d}=\int d^{d} x\left\langle j_{0}(x, t) j_{0}(0,0)\right\rangle \sim|t|^{\alpha-1},
$$

with

$$
\alpha=1-d / z=(2-d) /(2+d) .
$$

Equation (6) has to be integrated over $t$ to obtain $\kappa$ from Eq. (3). For a system of linear dimension $L$, the tail of $C_{0}(t)$ obtained in Eq. (6) is valid only when a disturbance at $x=0, t=0$ in the propagating modes has not been carried outside the system. This is because the tail in $\langle h(x, t) v(x, t) h(0,0) v(0,0)\rangle$ comes from long wavelength fluctuations, for which the contribution to $v$ from the heat diffusion mode is zero, so that $v$ must be expressed as a linear combination of the two propagating modes. A fluctuation in $v$ is carried to the boundaries of the system in a time $t \sim O(L)$. The precise behavior thereafter depends on the coupling to the heat baths at the boundaries, but in any case the fluctuation is partially or fully lost to the baths. The tail of $C_{0}(t)$ is cut off in a few round trip times, i.e., in a time $t \sim O(L)$. Substituting Eq. (6) into Eq. (3) and using this cutoff, we obtain

$$
\kappa(L)=L^{\alpha} .
$$

Thus the conductivity measured in a system of size $L$ diverges with $L$, or equivalently, the heat current flowing across a system with a fixed small temperature difference decays as $\sim L^{\alpha-1}$ with $L$. For the physically relevant case of $d=1$, the heat current must decay as $\sim L^{-2 / 3}$. For $d=2$, the conductivity $\kappa$ has a logarithmic singularity as a function of $L$.
We now compare with earlier analytical and numerical results. For a chain of coupled harmonic oscillators, there has been a large amount of analytical work showing that $\kappa$ diverges with system size [2]. The form of the divergence is different for different models, and in fact varies over a wide range depending on the heat baths [3]. However, all these are systems where local thermal equilibrium is not established, so the results of this paper do not apply. With more complicated models, there have been various numerical simulations that have shown a divergent $\kappa$, with $\alpha$ ranging from 0.17 to 0.5 [4-6]. The simulations in these papers were performed for fairly small system sizes, up to $\sim 1000$. The scaling of $\kappa$ as a function of $L$ is not very good, indicating the need for larger system sizes. However, recent simulations on large Fermi Pasta Ulam (FPU) chains [1,25], of size $\sim 10000$, have yielded $\alpha>0.36$, which disagrees with our calculation. We speculate that the discrepancy between the FPU numerics and our results are probably due to crossovers in the latter from the hydrodynamic modes of a crystal at intermediate length scales to those of a fluid (due to the absence of long-range order for $d=1$ ), but more work is needed to validate this.

Very recently, there have also been simulations on a one-dimensional hard sphere gas with alternating masses for much larger system sizes: up to 16383 [8] and 30000 [7]. The former estimates $\alpha$ to be 0.31 to 0.35 , and argues for an exact value of $1 / 3$ using the same scaling analysis as ours, but using the Burgers equation rather than the full equations of compressible 1D hydrodynamics. The latter, by a different analysis and with a different definition of the energy current, estimates $\alpha$ to be 0.255 . Further work is required to obtain a definite numerical value for $\alpha$ and to relate the energy currents defined in $[7,8]$ to the present paper.

In Ref. [4], there is a mode-coupling calculation that relies on Ref. [26], indicating that $\alpha$ should be $2 / 5$. The argument in [26] is internally inconsistent: the scaling $\omega \sim D(\omega) k^{2}$ is used to correctly find the renormalized diffusion coefficient $D(\omega) \sim \omega^{-1 / 3}$ and thereby the longtime tail of $C_{0}(t)$ as $\sim t^{-2 / 3}$, but then $\omega \sim k$ is used to incorrectly convert $D(\omega)$ to $D(k) \sim k^{-1 / 3}$. This expression is then used by Ref. [4] to obtain $C_{0}(t) \sim t^{-3 / 5}$. As we have seen, although $t \sim x$ is the scaling conversion to be used in Eq. (3), this is not appropriate for the loop integrals or the dynamic exponent. Second, although their system is nominally a 1D crystal, it should behave like a fluid at large length scales since fluctuations will wipe out long-range order. Nonetheless, if we treat it as a crystal, we will encounter the "Poisson-bracket" nonlinearity $\nabla u \delta H / \delta u$ in the equation for the momentum density, where $H$ is the elastic Hamiltonian for the displacement field $u$. This term is nonlinear even if we retain only harmonic terms in $H$. Power counting shows that this yields nonlinear corrections of the same type as those from the advective term. Apart from the above inconsistency the analysis of Ref. [4] is equivalent to this. 
Recent numerical studies on chains of coupled rotors [27] of length $L$ with angular-momentum conserving dynamics show a conductivity $\kappa(L)$ which saturates for large $L$ at high temperatures $T$. The inter-rotor potential is $1-\cos \left(x_{i+1}-x_{i}\right)$, and the rotors at the end are in contact with different heat baths. At high temperatures, since the elastic interaction between the rotators is effectively very weak, $x_{i+1}-x_{i}$ can be very large. If-by analogy to FPU chains - we interpret the $x_{i}$ s as displacements of particles from lattice sites, the "particle positions" are shuffled. Unlike for FPU chains, even at long wavelengths a localized disturbance is not advected outwards in $x$, and no anomaly is expected. However, to settle this issue completely will require a full nonlinear fluctuating hydrodynamic theory of the rotor lattice.

For a system in which momentum is not conserved, there is no advective term in the energy or mass current both of which depend on gradients of $\rho$ and $\epsilon$. Even the nonlinear terms in the equations for $\partial_{t} \rho$ and $\partial_{t} \epsilon$ thus have at least two spatial derivatives, and are irrelevant compared to the linear terms. The conductivity is given by Eq. (3) with $C(t)$ instead of $C_{0}(t)[14,16]$. This neither has a nonzero $C(\infty)$ limit, nor a slowly decaying tail for large $t$. Indeed, numerical studies [28] confirm that for such systems, the conductivity is finite. We note that if a system is integrable, even if it does not conserve momentum, the conductivity can be singular, since Refs. [14,16] assume local thermal equilibrium.

In this paper, we have shown that for one-dimensional momentum conserving systems, the heat current when a small temperature difference $\delta T$ is applied across a system of length $L$ is generically of the form $j \propto(\delta T) / L^{2 / 3}$. This is consistent with earlier numerical studies, but further work is needed to improve the numerical picture.

We thank Abhishek Dhar and Sriram Shastry for very useful comments and discussions.

*Present address.

[1] For a recent review of low-dimensional heat conduction, see S. Lepri, R. Livi, and A. Politi, cond-mat/0112193.

[2] Z. Rieder, J. L. Lebowitz, and E. Lieb, J. Math. Phys. (N.Y.) 8, 1073 (1967); A. Casher and J. L. Lebowitz, J. Math. Phys. (N.Y.) 12, 1701 (1971); R. J. Rubin and W. L. Greer, J. Math. Phys. (N.Y.) 12, 1686 (1971); A. J. O'Connor and J. L. Lebowitz, J. Math. Phys. (N.Y.) 15, 692 (1974); H. Spohn and J. L. Lebowitz, Commun. Math. Phys. 54, 97 (1977); H. Matsuda and K. Ishii, Prog.Theor. Phys.Suppl. 45, 56 (1970).

[3] A. Dhar, Phys. Rev. Lett. 86, 5882 (2001).

[4] S. Lepri, R. Livi, and A. Politi, Europhys. Lett. 43, 271 (1998).

[5] S. Lepri, R. Livi, and A. Politi, Phys. Rev. Lett. 78, 1896 (1997); A. V. Savin, G. P. Tsironis, and A. V. Zolotaryuk, Phys. Rev. Lett. 88, 154301 (2002); T. Hatano, Phys. Rev. E 59, R1 (1999).

[6] A. Dhar, Phys. Rev. Lett. 86, 3554 (2001).
[7] G. Casati and T. Prosen, cond-mat/0203331.

[8] P. Grassberger, W. Nadler, and L. Yang, Phys. Rev. Lett. 89, 180601 (2002).

[9] T. Prosen and D. K. Campbell, Phys. Rev. Lett. 84, 2857 (2000).

[10] M. S. Green, J. Chem. Phys. 22, 398 (1954).

[11] R. Kubo, J. Phys. Soc. Jpn. 12, 570 (1957).

[12] F. Bonetto, J. L. Lebowitz, and L. Rey-Bellet, math-ph/ 0002052.

[13] Alternatively, this can be written as the autocorrelation function of $J(t)$ restricted to the sector of the canonical ensemble in which $w$ is zero.

[14] J. A. McLennan, Non-Equilibrium Statistical Mechanics (Prentice Hall, Engelwood Cliffs, New Jersey, 1989).

[15] L. P. Kadanoff and P. C. Martin, Ann. Phys. (N.Y.) 24, 419 (1963).

[16] J. M. Luttinger, Phys. Rev. 135, A1505 (1964). Although this paper neglects thermal noise in the transport equations when it explicitly calculates transport coefficients, it is illuminating, since it shows how the correlation function defined with an $\omega \rightarrow 0 k \rightarrow 0$ limit for a closed system is related to the transport coefficients defined for a finite and open system in steady state, i.e., $\omega \rightarrow 0$ first. The equivalence between the two has to be verified, and is valid for momentum conserving systems only if $C(\infty)$ is subtracted from the correlation function. For instance, the derivation of the Kubo formula in Ref. [17] cited in Ref. [7] misses this issue.

[17] W. M. Visscher, Phys. Rev. A 10, 2461 (1974).

[18] D. Forster, Hydrodynamic Fluctuations, Broken Symmetry, and Correlation Functions (Benjamin Cummings, Reading, MA, 1975), Chap. 4.

[19] The propagating parts of the hydrodynamic modes do not affect this argument when the contribution of the same mode to $\langle h h\rangle$ and $\langle v v\rangle$ is considered, as can be seen by going to a moving frame. When different hydrodynamic modes are taken, the decay is $\sim t^{-d}$.

[20] Even in $d=1$ one expects a hydrodynamic description to be valid on long length scales for any nonintegrable system, although the length scale beyond which it applies could of course be very large.

[21] L. D. Landau and E. M. Lifshitz, Fluid Mechanics (Pergamon, Oxford, 1959).

[22] The most complete stochastic treatment of a fluid with temperature fluctuations is that of P. Español, Physica (Amsterdam) 248A, 77 (1998); see also W. van Saarloos and D. Bedeaux, Physica (Amsterdam) 110A, 147 (1982); D. N. Zubarev and V.G. Morozov, Physica (Amsterdam) 120A, 411 (1983).

[23] D. Forster, D. R. Nelson, and M. J. Stephen, Phys. Rev. A 16, 732 (1977).

[24] P. C. Martin, E. D. Siggia, and H. Rose, Phys. Rev. A 8, 423 (1973); R. Bausch, H. Wagner, and H. K. Janssen, Z. Phys. B 24, 113 (1976).

[25] P. Grassberger and L. Yang, cond-mat/0204247.

[26] M. H. Ernst, Physica (Amsterdam) 47D, 198 (1991).

[27] C. Giardina, R. Livi, A. Politi, and M. Vassalli, Phys. Rev. Lett. 84, 2144 (2000); O.V. Gendelman and A.V. Savin, Phys. Rev. Lett. 84, 2381 (2000).

[28] G. Casati, J. Ford, F. Vivaldi, and W. M. Visscher, Phys. Rev. Lett. 52, 1861 (1984); B. Hu, B. Li and H. Zhao, Phys. Rev. E 57, 2992 (1998). 